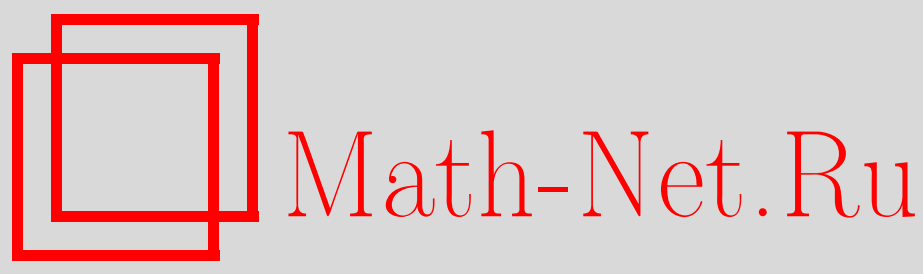

А. И. Аллилуева, А. И. Шафаревич, Нестандартные характеристики и локализованные асимптотические решения линеаризованной системы магнитной гидродинамики с малыми вязкостью и сопротивлением, ТМФ, 2017, том 190, номер 1, 191-204

DOI: https://doi.org/10.4213/tmf9228

Использование Общероссийского математического портала Math-Net.Ru подразумевает, что вы прочитали и согласны с пользовательским соглашением http://www.mathnet.ru/rus/agreement

Параметры загрузки:

IP: 54.89 .56 .158

26 апреля 2023 г., 16:50:09

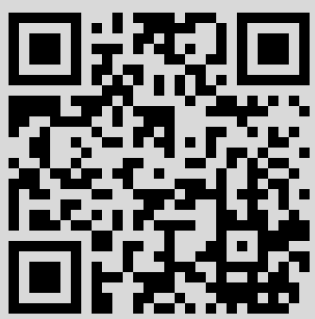




\section{НЕСТАНДАРТНЫЕ ХАРАКТЕРИСТИКИ И ЛОКАЛИЗОВАННЫЕ АСИМПТОТИЧЕСКИЕ РЕШЕНИЯ ЛИНЕАРИЗОВАННОЙ СИСТЕМЫ МАГНИТНОЙ ГИДРОДИНАМИКИ С МАЛЫМИ ВЯЗКОСТЬЮ И СОПРОТИВЛЕНИЕМ}

Описаны асимптотические решения задачи Коши для линеаризованной системы магнитной гидродинамики с начальными условиями, локализованными в малой окрестности кривой или двумерной поверхности. Исследовано влияние эффекта смены кратности характеристик на такие решения. Доказана равномерная оценка остатка.

Ключевые слова: уравнения магнитной гидродинамики, нестандартные характеристики.

DOI: $10.4213 / \operatorname{tmf9228}$

\section{1. ВВЕДЕНИЕ}

Линеаризованные уравнения магнитной гидродинамики (МГД) описывают эволюцию малых возмущений поля скоростей и магнитного поля в проводящей жидкости. Исследованию такой эволюции в разных ситуациях посвящена обширная литература (см., например, [1]-[3] и цитированную там литературу). При описании коротковолновых асимптотик решений этих уравнений возникают эффекты, связанные со сменой кратности характеристик, которая в данной ситуации оказывается весьма специфической (не выполнены стандартные условия общего положения); в работе [4] эти эффекты описаны в случае идеальной и идеально проводящей

Работа выполнена при финансовой поддержке Российского научного фонда (грант № 16-11-10282).

${ }^{*}$ Институт проблем механики им. А. Ю. Ишлинского РАН, Москва, Россия. E-mail: esina_anna@list.ru

${ }^{\dagger}$ Московский физико-технический институт, Долгопрудный, Московская обл., Россия

†Национальный исследовательский центр "Курчатовский институт", Москва, Россия

$\S$ Московский государственный университет им. М. В. Ломоносова, Москва, Россия. E-mail:shafarev@yahoo.com 
жидкости для решений ВКБ. В то же время с точки зрения приложений интересны локализованные возмущения (вихри), образующие узкие нити и тонкие пленки. При описании таких решений, помимо упомянутого выше пересечения гладких характеристических поверхностей, возникает еще особая поверхность, связанная с негладкостью символа оператора МГД. Кроме того, поскольку сильные вязкость и сопротивление быстро гасят мелкомасштабные возмущения, локализованные решения могут существовать только при условии малости старшей по производным части оператора. Другими словами, локализованные асимптотические решения связаны с нестандартными (в смысле В.П. Маслова [5]) характеристиками.

Ниже описана и обоснована асимптотика решения задачи Коши для уравнений МГД с малыми вязкостью и сопротивлением и с начальными условиями, локализованными вблизи кривой или двумерной поверхности. Решение представляется в виде суммы альфвеновских мод и слагаемых, описывающих их взаимодействие.

\section{2. ПОСТАНОВКА ЗАДАЧИ}

Малые возмущения заданного движения проводящей жидкости описываются линеаризованной системой МГД

$$
\begin{gathered}
\frac{\partial v}{\partial t}+(V, \nabla) v+(v, \nabla) V-(B, \nabla) b-(b, \nabla) B+\nabla P=\varepsilon^{2} \nu \triangle v \\
\frac{\partial b}{\partial t}+(V, \nabla) b-(b, \nabla) V+(v, \nabla) B-(B, \nabla) v=\varepsilon^{2} \mu \triangle b \\
(\nabla, v)=0, \quad(\nabla, b)=0 .
\end{gathered}
$$

Здесь $x \in \mathbb{R}^{3}, B(x), V(x)$ - заданные гладкие векторные поля (невозмущенные магнитное поле и поле скоростей), ограниченные вместе со всеми производными, $v, b$ - неизвестные векторные поля, зависящие от времени (возмущения поля скоростей и магнитного поля), $P$ - неизвестная скалярная функция (магнитное давление), $\mu, \nu$ - положительные константы (коэффициенты сопротивления и вязкости), $\varepsilon \rightarrow 0$ - малый параметр. Относительно полей $B, V$ будем предполагать, что они коммутируют:

$$
(V, \nabla) B-(B, \nabla) V=0
$$

это свойство выполнено, в частности, для стационарных решений нелинейной системы МГД. Для описания асимптотических решений удобно использовать сумму и разность внешних полей $U=V-B, W_{2}=V+B$ и самих решений $w=v-b$, $u=v+b$. В этих обозначениях уравнения приобретают вид

$$
\begin{gathered}
\frac{\partial u}{\partial t}+(W, \nabla) u+(w, \nabla) U+\nabla P=\varepsilon^{2}\left(\frac{\nu+\mu}{2} \Delta u+\frac{\nu-\mu}{2} \triangle w\right) \\
\frac{\partial w}{\partial t}+(U, \nabla) w+(u, \nabla) W+\nabla P=\varepsilon^{2}\left(\frac{\nu+\mu}{2} \Delta w+\frac{\nu-\mu}{2} \triangle u\right) \\
(\nabla, u)=0, \quad(\nabla, w)=0 .
\end{gathered}
$$


Сформулируем для этих уравнений задачу Коши с бездивергентными начальными условиями, локализованными вблизи кривой или поверхности:

$$
\left.u\right|_{t=0}=u_{0}\left(\frac{\Phi^{0}(x)}{\varepsilon}, x\right),\left.\quad w\right|_{t=0}=w_{0}\left(\frac{\Phi^{0}(x)}{\varepsilon}, x\right),
$$

где $u_{0}(y, x), w_{0}(y, x)$ - гладкие функции, ограниченные вместе со всеми производными и убывающие при $|y| \rightarrow \infty$ быстрее любой степени $|y|, \Phi(x)$ - гладкая скалярная или двумерная векторная функция, $y=\Phi(x) / \varepsilon$ - соответствующие “быстрые” переменные. Мы рассмотрим следующие две ситуации.

1. Начальные условия локализованы вблизи двумерной поверхности; в этом случае $\Phi^{0}$ - скалярная функция, причем уравнение $\Phi^{0}=0$ задает гладкую регулярную компактную двумерную поверхность $M_{0}$; мы будем считать, что длина вектора $\nabla \Phi^{0}$ ограничена сверху и снизу положительными константами.

2. Начальные условия локализованы вблизи двумерной кривой; в этом случае $\Phi^{0}$ - двумерная вектор-функция, причем уравнения $\Phi^{0}=0$ задают гладкую регулярную замкнутую кривую $\gamma_{0}$; мы будем считать, что векторы $\nabla \Phi_{1}^{0}, \nabla \Phi_{2}^{0}$ линейно независимы в $\mathbb{R}^{3}$ и постоянны вне компакта.

Наша цель - описание асимптотики решения задачи Коши (2), (3).

ЗАмЕчаниЕ 1. Из уравнений (2) можно исключить давление $P$; для этого рассмотрим проектор на бездивергентные векторные поля - псевдодифференциальный оператор $\widehat{\Pi}$ вида

$$
\widehat{\Pi} f=\frac{1}{(2 \pi)^{3 / 2}} \int_{\mathbb{R}^{3}} e^{i(k, x)} \Pi(k) \tilde{f} d k, \quad \Pi(k)=E-\frac{k \otimes k}{k^{2}},
$$

где $\tilde{f}$ - преобразование Фурье векторного поля $f, E$ - единичная матрица. Применяя этот оператор к уравнениям (2), получим

$$
\frac{\partial h}{\partial t}+\mathcal{L} h=\varepsilon^{2} \mathcal{D} \Delta h
$$

где

$$
h=\left(\begin{array}{c}
u \\
w
\end{array}\right), \quad \mathcal{L}=\left(\begin{array}{cc}
\widehat{\Pi}(W, \nabla) & \widehat{\Pi} \frac{\partial U}{\partial x} \\
\widehat{\Pi} \frac{\partial W}{\partial x} & \widehat{\Pi}(U, \nabla)
\end{array}\right), \quad \mathcal{D}=\frac{1}{2}\left(\begin{array}{cc}
\nu+\mu & \nu-\mu \\
\nu-\mu & \nu+\mu
\end{array}\right) .
$$

Используя тот факт, что коммутатор бездивергентного поля бездивергентен, все входящие в $\mathcal{L}$ дифференциальные операторы первого порядка можно вынести за оператор Пิ; в результате получим

$$
\mathcal{L}=\left(\begin{array}{cc}
\{W, \cdot\} & 0 \\
0 & \{U, \cdot\}
\end{array}\right)+\left(\begin{array}{cc}
\widehat{\Pi} \frac{\partial W}{\partial x} & \widehat{\Pi} \frac{\partial U}{\partial x} \\
\widehat{\Pi} \frac{\partial W}{\partial x} & \widehat{\Pi} \frac{\partial U}{\partial x}
\end{array}\right)
$$

где $\{\cdot, \cdot\}$ - коммутатор векторных полей. 


\section{3. ФОРМУЛИРОВКА РЕЗУЛЬТАТА}

Решение задачи Коши (2), (3) имеет следующую структуру. Асимптотический ряд состоит из слагаемых четырех типов: два из них описывают две альфвеновские моды, а два других - их взаимодействие, т. е. влияние первой моды на вторую и второй на первую. Опишем эти слагаемые. Введем сначала соответствующие характеристики. Пусть $g_{t}$ - поток поля $W$, а $q_{t}$ - поток поля $U$; рассмотрим фазовые функции

$$
\begin{aligned}
\Phi_{1}(x, t) & =\Phi^{0}\left(g_{-t} x\right), & \Phi_{2}(x, t) & =\Phi^{0}\left(q_{-t} x\right), \\
\Phi_{12}(x, t, \tau) & =\Phi^{0}\left(g_{-(t-\tau)} q_{-\tau} x\right), & \Phi_{21}(x, t, \tau) & =\Phi^{0}\left(q_{-(t-\tau)} g_{-\tau} x\right) .
\end{aligned}
$$

Отметим, что эти функции удовлетворяют уравнениям

$$
\begin{aligned}
\frac{\partial \Phi_{1}}{\partial t}+(W, \nabla) \Phi_{1} & =0, & \frac{\partial \Phi_{2}}{\partial t}+(U, \nabla) \Phi_{2} & =0 \\
\frac{\partial \Phi_{12}}{\partial t}+(W, \nabla) \Phi_{12} & =0, & \frac{\partial \Phi_{21}}{\partial t}+(U, \nabla) \Phi_{21} & =0
\end{aligned}
$$

и начальным условиям

$$
\left.\Phi_{j}\right|_{t=0}=\Phi^{0}(x),\left.\quad \Phi_{12}\right|_{t=\tau}=\Phi_{2}(\tau),\left.\quad \Phi_{21}\right|_{t=\tau}=\Phi_{1}(\tau)
$$

Отметим, кроме того, равенства (см. [4])

$$
\frac{\partial \Phi_{12}}{\partial \tau}=(W-U, \nabla) \Phi_{12}, \quad \frac{\partial \Phi_{21}}{\partial \tau}=(U-W, \nabla) \Phi_{21}
$$

Далее рассмотрим кокасательные векторы к $\mathbb{R}^{3}$, движущиеся вдоль соответствующих траекторий:

$$
p_{j}=\nabla \Phi_{j} k, \quad p_{i j}=\nabla \Phi_{i j} k
$$

где $k$ - число, если функция $\Phi_{0}$ скалярна (т. е. начальное условие локализовано вблизи поверхности), или двумерный вектор, если функция $\Phi_{0}$ векторная (начальное условие сосредоточено вблизи кривой; в этом случае $\nabla \Phi k-$ произведение $(3 \times 2)$-матрицы на двумерный вектор). Эти векторы удовлетворяют уравнениям

$$
\begin{aligned}
\dot{p}_{1}+\frac{\partial W^{*}}{\partial x} p_{1} & =0, & \dot{p}_{2}+\frac{\partial U^{*}}{\partial x} p_{2} & =0 \\
\dot{p}_{12}+\frac{\partial W^{*}}{\partial x} p_{12} & =0, & \dot{p}_{21}+\frac{\partial U^{*}}{\partial x} p_{21} & =0
\end{aligned}
$$

где точкой обозначена производная вдоль соответствующего поля ( $W$ или $U)$. 
Наконец, опишем амплитуды альфвеновских волн. Обозначим через $\widetilde{u}_{1}(x, t, k)$, $\widetilde{w}_{2}(x, t, k), \widetilde{u}_{12}(x, \xi, \eta, k), \widetilde{u}_{21}(x, \xi, \eta, k), \widetilde{w}_{12}(x, \xi, \eta, k), \widetilde{w}_{21}(x, \xi, \eta, k)$ решения следующих задач:

$$
\begin{aligned}
& \frac{\partial \widetilde{u}_{1}}{\partial t}+(W, \nabla) \widetilde{u}_{1}-\frac{p_{1} \otimes p_{1}}{p_{1}^{2}} \frac{\partial W}{\partial x} \widetilde{u}_{1}=-p_{1}^{2} \frac{\nu+\mu}{2} \widetilde{u}_{1},\left.\quad \widetilde{u}_{1}\right|_{t=0}=\widetilde{u}_{0}(k, x) ; \\
& \frac{\partial \widetilde{w}_{2}}{\partial t}+(U, \nabla) \widetilde{w}_{2}-\frac{p_{2} \otimes p_{2}}{p_{2}^{2}} \frac{\partial U}{\partial x} \widetilde{w}_{2}=-p_{2}^{2} \frac{\nu+\mu}{2} \widetilde{w}_{2},\left.\quad \widetilde{w}_{2}\right|_{t=0}=\widetilde{w}_{0}(k, x) ; \\
& \frac{\partial \widetilde{u}_{12}}{\partial \xi}+(W, \nabla) \widetilde{u}_{12}+\frac{\partial U}{\partial x} \widetilde{w}_{12}-\frac{p_{12} \otimes p_{12}}{p_{12}^{2}}\left(\frac{\partial U}{\partial x} \widetilde{w}_{12}+\frac{\partial W}{\partial x} \widetilde{u}_{12}\right)= \\
& =-p_{12}^{2}\left(\frac{\nu+\mu}{2} u_{12}+\frac{\nu-\mu}{2} \widetilde{w}_{12}\right) \text {, } \\
& \frac{\partial \widetilde{w}_{12}}{\partial \eta}+(U, \nabla) \widetilde{w}_{12}+\frac{\partial W}{\partial x} \widetilde{u}_{12}-\frac{p_{12} \otimes p_{12}}{p_{12}^{2}}\left(\frac{\partial U}{\partial x} \widetilde{w}_{12}+\frac{\partial W}{\partial x} \widetilde{u}_{12}\right)= \\
& =-p_{12}^{2}\left(\frac{\nu+\mu}{2} \widetilde{w}_{12}+\frac{\nu-\mu}{2} \widetilde{u}_{12}\right) \text {, } \\
& \left.\widetilde{w}_{12}\right|_{\eta=0}=0,\left.\quad \widetilde{u}_{12}\right|_{\xi=0}=-\left(p_{2}^{2} \frac{\nu-\mu}{2}+\left(E-\frac{p_{2} \otimes p_{2}}{p_{2}^{2}}\right) \frac{\partial U}{\partial x}\right) \widetilde{w}_{2} ; \\
& \frac{\partial \widetilde{u}_{21}}{\partial \xi}+(W, \nabla) \widetilde{u}_{21}+\frac{\partial U}{\partial x} \widetilde{w}_{21}-\frac{p_{21} \otimes p_{21}}{p_{21}^{2}}\left(\frac{\partial U}{\partial x} \widetilde{w}_{21}+\frac{\partial W}{\partial x} \widetilde{u}_{21}\right)= \\
& =-p_{21}^{2}\left(\frac{\nu+\mu}{2} \widetilde{u}_{21}+\frac{\nu-\mu}{2} \widetilde{w}_{21}\right) \text {, } \\
& \frac{\partial \widetilde{w}_{21}}{\partial \eta}+(U, \nabla) \widetilde{w}_{21}+\frac{\partial W}{\partial x} \widetilde{u}_{21}-\frac{p_{21} \otimes p_{21}}{p_{21}^{2}}\left(\frac{\partial U}{\partial x} \widetilde{w}_{21}+\frac{\partial W}{\partial x} \widetilde{u}_{21}\right)= \\
& =-p_{21}^{2}\left(\frac{\nu+\mu}{2} \widetilde{w}_{21}+\frac{\nu-\mu}{2} \widetilde{u}_{21}\right) \text {, } \\
& \left.\widetilde{u}_{21}\right|_{\eta=0}=0,\left.\quad \widetilde{w}_{21}\right|_{\xi=0}=-\left(p_{1}^{2} \frac{\nu-\mu}{2}+\left(E-\frac{p_{1} \otimes p_{1}}{p_{1}^{2}}\right) \frac{\partial U}{\partial x}\right) \widetilde{u}_{1} .
\end{aligned}
$$

Здесь $\widetilde{u}_{0}(x, k), \widetilde{w}_{0}(x, k)$ - преобразования Фурье начальных функций по “быстрым" переменным $y$.

ЗАмЕчАниЕ 2. Задачи (12), (13) после перехода на характеристики сводятся к задачам Коши для линейных обыкновенных дифференциальных уравнений. Задачи (14), (15) аналогичны задаче Гурса - их разрешимость и гладкость устанавливается сведением к интегральным уравнениям и применением метода последовательных приближений. 
Основной результат работы - следующее утверждение.

Теорема 1. Решение задачи Коши (2), (3) представляется в виде

$$
\begin{aligned}
& u=\frac{1}{(2 \pi)^{s / 2}} \int_{\mathbb{R}^{s}} e^{(i / \varepsilon) k \cdot \Phi_{1}(x, t)} \widetilde{u}_{1}(x, t, k) d k+ \\
& +\left.\int_{0}^{t} d \tau \frac{1}{(2 \pi)^{s / 2}} \int_{\mathbb{R}^{s}} e^{(i / \varepsilon) k \cdot \Phi_{12}(x, t, \tau)} \widetilde{u}_{12}(x, \xi, \eta, k)\right|_{\xi=t-\tau, \eta=\tau} d k+ \\
& +\left.\int_{0}^{t} d \tau \frac{1}{(2 \pi)^{s / 2}} \int_{\mathbb{R}^{s}} e^{(i / \varepsilon) k \cdot \Phi_{21}(x, t, \tau)} \widetilde{u}_{21}(x, \xi, \eta, k)\right|_{\xi=t-\tau, \eta=\tau} d k+\widehat{u}, \quad \widehat{u}=O\left(\varepsilon^{1-\alpha}\right), \\
& w=\frac{1}{(2 \pi)^{s / 2}} \int_{\mathbb{R}^{s}} e^{(i / \varepsilon) k \cdot \Phi_{2}(x, t)} \widetilde{w}_{2}(x, t, k) d k+ \\
& +\left.\int_{0}^{t} d \tau \frac{1}{(2 \pi)^{s / 2}} \int_{\mathbb{R}^{s}} e^{(i / \varepsilon) k \cdot \Phi_{12}(x, t, \tau)} \widetilde{w}_{12}(x, \xi, \eta, k)\right|_{\xi=t-\tau, \eta=\tau} d k+ \\
& +\left.\int_{0}^{t} d \tau \frac{1}{(2 \pi)^{s / 2}} \int_{\mathbb{R}^{s}} e^{(i / \varepsilon) k \cdot \Phi_{21}(x, t, \tau)} \widetilde{w}_{21}(x, \xi, \eta, k)\right|_{\xi=t-\tau, \eta=\tau} d k+\widehat{w}, \quad \widehat{w}=O\left(\varepsilon^{1-\alpha}\right) .
\end{aligned}
$$

Здесь $s=2$ для решений, локализованных вблизи поверхности кривой, $u s=1$ для решений, локализованных вблизи поверхности, $\alpha>0$ может быть сделано сколь угодно малым (не зависящим от $\varepsilon$ ), оценка остатка понимается в равномерной норме.

ЗАмЕчАниЕ 3. Первые слагаемые в выражениях для каждой функции представляют собой альфвеновские моды, порожденные начальными локализованными полями. Слагаемые, содержащие интегралы по $\tau$, описывают взаимодействие альфвеновских мод.

ЗАмЕчАниЕ 4. Очевидно, альфвеновские моды локализованы вблизи сдвига начального асимптотического носителя (поверхности $M_{0}$ или кривой $\gamma_{0}$ ) вдоль траекторий поля $W$ или $U$, соответствующая гладкая поверхность или кривая задается уравнениями $\Phi_{1}(x, t)=0$ или $\Phi_{2}(x, t)=0$. Локализация слагаемых, описывающих взаимодействие, вообще говоря, может быть различной, она зависит от наличия и структуры точек касания начального асимптотического носителя. В дальнейшем мы планируем детально исследовать локализацию и возможность временно́го роста асимптотических решений.

Ниже приведено конструктивное доказательство теоремы 1.

\section{4. КОНСТРУКЦИЯ ФОРМАЛЬНОЙ АСИМПТОТИКИ}

4.1. Формальный ряд для регуляризованной задачи. Для определенности будем считать, что $s=2$ (решение локализовано в окрестности кривой); для $s=1$ все выкладки несколько проще. Поскольку разложение локализованной функции содержит, вообще говоря, все гармоники, а символ оператора (4) негладкий при нулевых кокасательных векторах, для построения асимптотического ряда нужно вырезать малую окрестность нулевых гармоник и затем отдельно оценить образовавшуюся функцию. С этой целью введем срезающую функцию $\chi(k), k \in \mathbb{R}^{s}$, 
равную нулю в окрестности нуля и единице в окрестности бесконечности; разобьем начальное условие $h^{0}={ }^{t}\left(u^{0}, w^{0}\right)$ на две части:

$$
h^{0}(y, x, t)=h_{A}^{0}+h_{B}^{0}, \quad h_{A}^{0}=\frac{1}{(2 \pi)^{s / 2}} \int_{\mathbb{R}^{s}} e^{i \Phi^{0} \cdot k / \varepsilon} \chi\left(\frac{k}{\varepsilon^{\beta}}\right) \widetilde{h}^{0}(k, x) d k,
$$

где $\beta \in(0,1)$ и достаточно близко к единице. Сначала заменим начальное поле $h^{0}$ на $h_{A}^{0}$. Будем искать формальное асимптотическое решение этой задачи в виде

$$
h(x, t, \varepsilon)=\sum_{j=0}^{\infty} \varepsilon^{j}\left(h_{1}^{j}+h_{2}^{j}+h_{12}^{j}+h_{21}^{j}\right),
$$

где

$$
\begin{aligned}
h_{l}^{j} & =\frac{1}{(2 \pi)^{s / 2}} \int_{\mathbb{R}^{s}} e^{(i / \varepsilon) k \cdot \Phi_{l}(x, t)} \widetilde{h}_{l}^{j}(k, x, t) \chi\left(\frac{k}{\varepsilon^{\beta}}\right) d k, \\
h_{l m} & =\int_{0}^{t} d \tau \frac{1}{(2 \pi)^{s / 2}} \int_{\mathbb{R}^{s}} e^{(i / \varepsilon) k \cdot \Phi_{l m}(x, t, \tau)} \widetilde{h}_{l m}^{j}(x, t, \tau, k) \chi\left(\frac{k}{\varepsilon^{\beta}}\right) d k .
\end{aligned}
$$

В аналогичном виде будем искать давление (соответствующие слагаемые обозначим $P_{l}^{j}$ и $\left.P_{l m}^{j}\right)$. Подставим (18) в $(2)$ и приравняем слагаемые при разных степенях $\varepsilon$. При $\varepsilon^{-1}$ вне интегралов по $\tau$ получим

$$
\left(\begin{array}{cc}
\frac{\partial \Phi_{l}}{\partial t}+(W, \nabla) \Phi_{l} & 0 \\
0 & \frac{\partial \Phi_{l}}{\partial t}+(U, \nabla) \Phi_{l}
\end{array}\right) \cdot \frac{\partial}{\partial y} h_{l}^{0}+\left(\begin{array}{c}
\nabla \Phi_{l}^{0} \\
\nabla \Phi_{l}
\end{array}\right) \cdot \frac{\partial}{\partial y} P_{l}=0
$$

Из условий $(\nabla, u)=0,(\nabla, w)=0$ вытекает, что $\left(\nabla \Phi_{l}, \nabla\right) \cdot\left(\partial h^{0} / \partial y\right)=0$. Умножая вектор из первых трех строк в (19) скалярно на градиенты функций $\Phi_{l}^{m}$ (компонент вектора $\left.\Phi_{l}\right)$, получим

$$
\sum_{m, n}\left(\nabla \Phi_{l}^{m}, \nabla \Phi_{l}^{n}\right) \frac{\partial^{2}}{\partial y_{m} \partial y_{n}} P_{l}^{0}=0
$$

Это эллиптическое уравнение, поэтому из условия убывания $P_{l}$ на бесконечности вытекает, что $P_{l}=0$. Теперь из равенств $(19)$ вытекают уравнения $(7)$ на $\Phi_{l}$ и структура векторов $h_{l}^{0}$ :

$$
\frac{\partial \Phi_{1}}{\partial t}+(W, \nabla) \Phi_{1}=0, \quad \frac{\partial \Phi_{2}}{\partial t}+(U, \nabla) \Phi_{2}=0, \quad w_{1}^{0}=0, \quad u_{2}^{0}=0 .
$$

В дальнейшем положим $w_{1}^{j}=0, u_{2}^{j}=0$ для всех $j$; ниже доказано существование формальной асимптотики с такой структурой. Рассмотрим теперь слагаемые того же порядка, содержащие интегралы по $\tau$ :

$$
\begin{aligned}
\sum_{k \neq m} \int_{0}^{t}( & \left.\left(\frac{\partial \Phi_{l m}}{\partial t}+(W, \nabla) \Phi_{l m}\right) \cdot \frac{\partial u_{l m}^{0}}{\partial y}+\nabla \Phi_{l m} \cdot \frac{\partial P_{l m}^{0}}{\partial y}\right) d \tau=O(\varepsilon) \\
\sum_{k \neq m} \int_{0}^{t}( & \left.\left(\frac{\partial \Phi_{l m}}{\partial t}+(U, \nabla) \Phi_{l m}\right) \cdot \frac{\partial w_{l m}^{0}}{\partial y}+\nabla \Phi_{l m} \cdot \frac{\partial P_{l m}^{0}}{\partial y}\right) d \tau=O(\varepsilon), \\
\int_{0}^{t} \frac{\partial}{\partial y} \cdot\left(u_{l m}^{0}, \nabla\right) \Phi_{l m} d \tau=\int_{0}^{t} \frac{\partial}{\partial y} \cdot\left(w_{l m}^{0}, \nabla\right) \Phi_{l m} d \tau & =O(\varepsilon) .
\end{aligned}
$$


Учитывая уравнения (7) на функции $\Phi_{l m}$ и полагая $P_{l m}^{0}=0$, получим, что векторные равенства приводятся к виду

$$
\begin{aligned}
& \int_{0}^{t}\left(\frac{\partial \Phi_{21}}{\partial t}+(W, \nabla) \Phi_{21}\right) \cdot \frac{\partial}{\partial y} u_{21}^{0} d \tau=O(\varepsilon), \\
& \int_{0}^{t}\left(\frac{\partial \Phi_{12}}{\partial t}+(U, \nabla) \Phi_{12}\right) \cdot \frac{\partial}{\partial y} w_{12}^{0} d \tau=O(\varepsilon) .
\end{aligned}
$$

Преобразуем первый интеграл:

$$
\begin{aligned}
& \int_{0}^{t}\left(\frac{\partial \Phi_{21}}{\partial t}+(W, \nabla) \Phi_{21}\right) \cdot \frac{\partial}{\partial y} u_{21}^{0} d \tau= \\
& =\int_{0}^{t}\left(\frac{\partial \Phi_{21}}{\partial t}+(U, \nabla) \Phi_{21}-(U-W, \nabla) \Phi_{21}\right) \cdot \frac{\partial}{\partial y} u_{21}^{0} d \tau=-\int_{0}^{t} \frac{\partial \Phi_{21}}{\partial \tau} \cdot \frac{\partial}{\partial y} u_{21}^{0} d \tau= \\
& =-\varepsilon \int_{0}^{t}\left(\frac{d}{d \tau} u_{21}^{0}\left(\frac{\Phi_{21}(x, t, \tau)}{\varepsilon}, x, t, \tau\right)-\frac{\partial u_{21}^{0}}{\partial \tau}\left(\frac{\Phi_{21}(x, t, \tau)}{\varepsilon}, x, t, \tau\right)\right) d \tau= \\
& =-\left.\varepsilon u_{21}^{0}\right|_{\tau=t}+\varepsilon \int_{0}^{t} \frac{\partial u_{21}^{0}}{\partial \tau}\left(\frac{\Phi_{21}(x, t, \tau)}{\varepsilon}, x, t, \tau\right) d \tau .
\end{aligned}
$$

Здесь мы использовали равенства (9) и положили $\left.u_{21}^{0}\right|_{\tau=0}=0$ (ср. с (15)). Аналогично преобразуется второй интеграл в (21), в результате эти равенства принимают вид

$$
\begin{aligned}
& -\left.\varepsilon u_{21}^{0}\right|_{\tau=t}+\varepsilon \int_{0}^{t} \frac{\partial u_{21}^{0}}{\partial \tau}\left(\frac{\Phi_{21}(x, t, \tau)}{\varepsilon}, x, t, \tau\right) d \tau=O(\varepsilon), \\
& -\left.\varepsilon w_{12}^{0}\right|_{\tau=t}+\varepsilon \int_{0}^{t} \frac{\partial w_{12}^{0}}{\partial \tau}\left(\frac{\Phi_{12}(x, t, \tau)}{\varepsilon}, x, t, \tau\right) d \tau=O(\varepsilon) .
\end{aligned}
$$

Таким образом, свойства функций $\Phi_{j}, \Phi_{l m}$ гарантируют выполнение векторных равенств $(2)(\bmod O(1))$, причем в следующее приближение переходят слагаемые $(22)$.

Соберем теперь слагаемые при $\varepsilon^{0}$, возникающие при подстановке ряда (18) в уравнения (2). С учетом свойств функций $\Phi, h^{1}$ и равенств $(22)$ вне интегралов по $\tau$ получаем

$$
\begin{aligned}
\sum_{l} & {\left[\left(\begin{array}{cc}
\frac{\partial}{\partial t}+(W, \nabla) & \frac{\partial U}{\partial x} \\
\frac{\partial W}{\partial x} & \frac{\partial}{\partial t}+(U, \nabla)
\end{array}\right) h_{l}^{0}+\left(\begin{array}{c}
\nabla \Phi_{l}^{1} \\
\nabla \Phi_{l}^{1}
\end{array}\right) \cdot \frac{\partial}{\partial y} P_{l}^{1}\right]+} \\
& +\sum_{s \neq l}\left(\left.h_{s l}^{0}\right|_{\tau=t}+\left.h_{l s}^{0}\right|_{\tau=t}\right)=\left.\left(\begin{array}{c}
u_{21}^{0} \\
w_{12}^{0}
\end{array}\right)\right|_{t=\tau}+\sum_{m, n, l}\left(\nabla \Phi_{l}^{m}, \nabla \Phi_{l}^{n}\right) \frac{\partial^{2}}{\partial y_{m} \partial y_{n}} \mathcal{D} h_{l}^{0},
\end{aligned}
$$

где $s \neq l$. Здесь учтены внеинтегральные слагаемые в (22). Рассмотрим первое векторное уравнение (т. е. уравнение, содержащее $\partial u / \partial t)$, для второго выкладки аналогичны. Учитывая структуру вектора $h_{1}^{0}\left(w_{1}^{0}=0\right)$, получим

$$
\begin{aligned}
& \left(\frac{\partial}{\partial t}+(W, \nabla)\right) u_{1}^{0}+\nabla \Phi_{1} \cdot \frac{\partial}{\partial y} P_{1}^{1}-\frac{\mu+\nu}{2} \sum_{m, n}\left(\nabla \Phi_{1}^{m}, \nabla \Phi_{1}^{n}\right) \frac{\partial^{2}}{\partial y_{m} \partial y_{n}} u_{1}^{0}= \\
& =-\nabla \Phi_{2} \cdot \frac{\partial}{\partial y} P_{2}^{1}-\frac{\partial U}{\partial x} w_{2}^{0}-\left.u_{12}^{0}\right|_{\tau=t}+\frac{\nu-\mu}{2} \sum_{m, n}\left(\nabla \Phi_{2}^{m}, \nabla \Phi_{2}^{n}\right) \frac{\partial^{2}}{\partial y_{m} \partial y_{n}} w_{2}^{0} .
\end{aligned}
$$


Функции в левой части равенства зависят от быстрых переменных $\Phi_{1} / \varepsilon$, а в правой части - от быстрых переменных $\Phi_{2} / \varepsilon$ (напомним, что $\left.\left.\Phi_{21}\right|_{\tau=t}=\Phi_{2}(t)\right)$, поэтому обе части равенства должны обращаться в нуль. Рассмотрим сначала правую часть. Подставляя вместо функций от у соответствующие интегралы (см. (18)), получим

$$
i p_{2} \widetilde{P}_{2}^{1}+\frac{\partial U}{\partial x} \widetilde{w}_{2}^{0}+\left.\widetilde{u}_{12}^{0}\right|_{\tau=t}+\frac{\nu-\mu}{2} p_{2}^{2} \widetilde{w}_{2}^{0}=0 .
$$

Спроецируем это равенство на прямую, параллельную вектору $p_{2}$, и на ортогональную к ней плоскость; поскольку из условий $(\nabla, u)=0,(\nabla, w)=0$ следует, что $\left(p_{2}, \widetilde{w}_{2}^{0}\right)=0$ и $\left(p_{2},\left.\widetilde{u}_{21}\right|_{\tau=t} ^{0}\right)=0$, получаем

$$
\widetilde{P}_{2}^{1}=\frac{i}{p_{2}^{2}}\left(p_{2}, \frac{\partial U}{\partial x} \widetilde{w}_{2}^{0}\right)
$$

и

$$
\left.\widetilde{u}_{12}^{0}\right|_{\tau=t}=-\frac{\nu-\mu}{2} p_{2}^{2} \widetilde{w}_{2}^{0}-\left(E-\frac{p_{2} \otimes p_{2}}{p_{2}^{2}}\right) \frac{\partial U}{\partial x} \widetilde{w}_{2}^{0}
$$

(отметим, что последнее равенство и условие $\left.u_{21}^{0}\right|_{\tau=0}=0$ совпадают с условиями в (14), (15)).

Рассмотрим теперь левую часть в (24). Подставляя вместо функций от $y$ интегралы, получаем

$$
\left(\frac{\partial}{\partial t}+(W, \nabla)\right) \widetilde{u}_{1}^{0}+i p_{1} \widetilde{P}_{1}^{1}+p_{1}^{2} \frac{\mu+\nu}{2} \widetilde{u}_{1}^{0}=0
$$

Спроецируем это равенство на прямую, параллельную вектору $p_{1}$, и на ортогональную к ней плоскость; отметим, что, поскольку функция $\Phi_{1}$ удовлетворяет уравнениям $(7), p_{1}$ удовлетворяет (11). Кроме того, из условия $(\nabla, u)=0$ следует, что $\left(p_{1}, \widetilde{u}_{1}^{0}\right)=0$, поэтому

$$
\left(p_{1},\left(\frac{\partial}{\partial t}+(W, \nabla)\right) \widetilde{u}_{1}^{0}\right)=\left(\frac{\partial}{\partial t}+(W, \nabla)\right)\left(p_{1}, \widetilde{u}_{1}^{0}\right)-\left(u_{1}^{0}, \dot{p}_{1}\right)=\left(p_{1}, \frac{\partial W}{\partial x} \widetilde{u}_{1}^{0}\right) .
$$

В результате две указанные проекции приводят к равенствам

$$
\widetilde{P}_{1}^{1}=\frac{i}{p_{1}^{2}}\left(p_{1} \frac{\partial W}{\partial x} \widetilde{u}_{1}^{0}\right)
$$

И

$$
\frac{\partial \widetilde{u}_{1}^{0}}{\partial t}+(W, \nabla) \widetilde{u}_{1}^{0}-\frac{p_{1} \otimes p_{1}}{p_{1}^{2}} \frac{\partial W}{\partial x} \widetilde{u}_{1}^{0}=-p_{1}^{2} \frac{\nu+\mu}{2} \widetilde{u}_{1}^{0}
$$

отметим, что последнее уравнение совпадает с (12).

Аналогичные выкладки, примененные ко второму уравнению в (23), приводят к тем же равенствам $(26),(25)$, уравнению (13) для $\widetilde{w}_{2}^{0}$ и условиям $\left.\widetilde{w}_{12}^{0}\right|_{\tau=0}=0$ и

$$
\left.\widetilde{w}_{21}^{0}\right|_{\tau=t}=-\frac{\nu-\mu}{2} p_{1}^{2} \widetilde{u}_{1}^{0}-\left(E-\frac{p_{1} \otimes p_{1}}{p_{1}^{2}}\right) \frac{\partial W}{\partial x} \widetilde{u}_{1}^{0},
$$

совпадающим с условиями в (14), (15). 
Рассмотрим теперь слагаемые порядка $\varepsilon^{0}$, содержащие интегралы по $\tau$. Выпишем уравнения, содержащие функции с индексами 12 (для функций с индексами 21 выкладки аналогичны). Заменим функции от $y$ соответствующими интегралами; приравнивая к нулю подынтегральные функции, с учетом слагаемых (22) получаем

$$
\begin{array}{r}
\left(\frac{\partial}{\partial t}+(W, \nabla)\right) \widetilde{u}_{12}^{0}+\frac{\partial U}{\partial x} \widetilde{w}_{12}^{0}+i p_{12} P_{12}^{1}=-p_{12}^{2}\left(\frac{\nu+\mu}{2} \widetilde{u}_{12}^{0}+\frac{\nu-\mu}{2} \widetilde{w}_{12}^{0}\right) \\
\left(\frac{\partial}{\partial t}+(U, \nabla)\right) \widetilde{w}_{12}^{0}+\frac{\partial W}{\partial x} \widetilde{u}_{12}^{0}+i p_{12} P_{12}^{1}+\frac{\partial w_{12}^{0}}{\partial \tau}=-p_{12}^{2}\left(\frac{\nu+\mu}{2} \widetilde{w}_{12}^{0}+\frac{\nu-\mu}{2} \widetilde{u}_{12}^{0}\right) .
\end{array}
$$

Применим к этим уравнениям ту же процедуру проецирования на прямую, параллельную вектору $p_{12}$, и ортогональную ему плоскость. Отметим равенства

$$
\begin{aligned}
& \left(p_{12},\left(\frac{\partial}{\partial t}+(W, \nabla)\right) \widetilde{u}_{12}^{0}+\frac{\partial U}{\partial x} \widetilde{w}_{12}^{0}\right)= \\
& \quad=\left(\frac{\partial}{\partial t}+(W, \nabla)\right)\left(\widetilde{u}_{12}^{0}, p_{12}^{0}\right)+\left(p_{12}^{0}, \frac{\partial U}{\partial x} \widetilde{w}_{12}^{0}+\frac{\partial W}{\partial x} \widetilde{u}_{12}^{0}\right)= \\
& \quad=\left(p_{12}^{0}, \frac{\partial U}{\partial x} \widetilde{w}_{12}^{0}+\frac{\partial W}{\partial x} \widetilde{u}_{12}^{0}\right)
\end{aligned}
$$

и

$$
\begin{aligned}
\left(p_{12},\right. & \left.\left(\frac{\partial}{\partial t}+(U, \nabla)\right) \widetilde{w}_{12}^{0}+\frac{\partial W}{\partial x} \widetilde{u}_{12}^{0}+\frac{\partial \widetilde{w}_{12}^{0}}{\partial \tau}\right)= \\
= & \left(\frac{\partial}{\partial t}+(U, \nabla)+\frac{\partial}{\partial \tau}\right)\left(p_{12}, \widetilde{w}_{12}^{0}\right)-\left(\widetilde{w}_{12}^{0},\left(\frac{\partial}{\partial t}+(U, \nabla)+\frac{\partial}{\partial \tau}\right) p_{12}\right)+ \\
& +\left(p_{12}, \frac{\partial W}{x} \widetilde{u}_{12}^{0}\right)=\left(p_{12}, \frac{\partial U}{\partial x} \widetilde{w}_{12}^{0}+\frac{\partial W}{\partial x} \widetilde{u}_{12}^{0}\right)
\end{aligned}
$$

Эти равенства вытекают из формул (7), (9), (11), $\left(p_{12}, \widetilde{u}_{12}^{0}\right)=0,\left(p_{12}, \widetilde{w}_{12}^{0}\right)=0$ (последние, в свою очередь, следуют из условий $(\nabla, u)=0,(\nabla, w)=0)$. Из приведенных равенств немедленно получаем

$$
\widetilde{P}_{12}^{1}=\frac{i}{p_{12}^{2}}\left(p_{12}, \frac{\partial U}{\partial x} \widetilde{w}_{12}^{0}+\frac{\partial W}{\partial x} u_{12}^{0}\right)
$$

и

$$
\begin{aligned}
\frac{\partial \widetilde{u}_{12}^{0}}{\partial t} & +(W, \nabla) \widetilde{u}_{12}^{0}+\frac{\partial U}{\partial x} \widetilde{w}_{12}^{0}-\frac{p_{12} \otimes p_{12}}{p_{12}^{2}}\left(\frac{\partial U}{\partial x} \widetilde{w}_{12}^{0}+\frac{\partial W}{\partial x} u_{12}^{0}\right)= \\
& =-p_{12}^{2}\left(\frac{\nu+\mu}{2} \widetilde{u}_{12}^{0}+\frac{\nu-\mu}{2} \widetilde{w}_{12}^{0}\right), \\
\frac{\partial \widetilde{w}_{12}^{0}}{\partial t} & +\frac{\partial \widetilde{w}_{12}^{0}}{\partial \tau}+(U, \nabla) \widetilde{w}_{12}^{0}+\frac{\partial W}{\partial x} \widetilde{u}_{12}^{0}-\frac{p_{12} \otimes p_{12}}{p_{12}^{2}}\left(\frac{\partial U}{\partial x} \widetilde{w}_{12}^{0}+\frac{\partial W}{\partial x} u_{12}^{0}\right)= \\
& =-p_{12}^{2}\left(\frac{\nu+\mu}{2} \widetilde{w}_{12}^{0}+\frac{\nu-\mu}{2} \widetilde{u}_{12}^{0}\right) .
\end{aligned}
$$

Замена переменных $\xi=t-\tau, \eta=\tau$ приводит последние уравнения к виду (14). 
Аналогично получаются уравнения для функций $\widetilde{u}_{21}^{0}, \widetilde{w}_{21}^{0}, \widetilde{P}_{21}^{1}$; ясно, что все эти уравнения вместе с полученными ранее начальными условиями однозначно разрешимы (отметим, что задачи (14) и (15) аналогичны задачам Гурса). Если функции $\widetilde{u}_{1}^{0}, \widetilde{w}_{2}^{0}, \widetilde{u}_{l s}^{0}, \widetilde{w}_{l s}^{0}$ удовлетворяют выписанным задачам, ряд (18) удовлетворяет задаче Коши для системы МГД с начальным условием $h_{A}^{0}(\bmod O(\varepsilon))$; отметим, что указанные функции ограничены при $k \rightarrow 0$, в то время как функции $\widetilde{P}_{l}^{1}, \widetilde{P}_{l s}^{1}$ растут, как $1 /|k|$ (в соответствующем интеграле эта особенность убирается срезающей функцией $\chi)$.

Остальные слагаемые ряда (18) определяются по той же схеме; они удовлетворяют аналогичным уравнениям с правыми частями, причем легко понять, что при $k \rightarrow 0$ функции $\widetilde{u}_{1}^{j}, \widetilde{w}_{2}^{j}, \widetilde{u}_{l s}^{j}, \widetilde{w}_{l s}^{j}, \widetilde{P}_{l}^{j}, P_{l s}^{j}$ растут, как $O\left(|k|^{-j}\right)$. Сформулируем полученное утверждение.

Лемма 1. Существует формальный ряд (18), удовлетворяющий системе МГД с начальным условием $h_{A}^{0}$; при этом функции $\Phi_{l}, \Phi_{l m}, \widetilde{u}_{l}^{j}, \widetilde{w}_{l}^{j}, \widetilde{u}_{l m}^{j}, \widetilde{w}_{l m}^{j}, \widetilde{P}_{l}^{j}, \widetilde{P}_{l m}^{j}$ удовлетворяют задачам, выписанным в разделе 3 , с заменой начальных условий на функиии $h_{A}^{0}$ (при $j \geqslant 1$ у систем имеются правые части, а начальные условия нулевые). При $k \rightarrow 0$ указанные функиии растут, как $O\left(|k|^{-j}\right)$.

ЗАмЕчАниЕ 5. Срезающая функция $\chi$ предотвращает расходимость интегралов, входящих в слагаемые ряда (18), но влияет на их оценку. Легко понять, что нормы этих слагаемых в пространствах Соболева оцениваются следующим образом:

$$
\left\|h_{l}^{j}\right\|_{W_{2}^{m}}\left(\mathbb{R}^{3}\right)=O\left(\varepsilon^{(j-m)(1-\beta)+s / 2}\right),
$$

причем $N$-я частичная сумма $H_{N}$ этого ряда удовлетворяет задаче

$$
\frac{\partial H_{N}}{\partial t}+\mathcal{L} H_{N}=\varepsilon^{2} \mathcal{D} \Delta H_{N}+F_{N},\left.\quad H_{N}\right|_{t=0}=h_{A}^{0},
$$

где $\left\|F_{N}\right\|_{W_{2}^{m}}=O\left(\varepsilon^{(N+1-m)(1-\beta)+s / 2}\right)$.

4.2. Оценка поля $h_{B}^{0}$. Из разложения (17) начального поля вытекает следующая формула для $h_{B}^{0}$ :

$$
h_{B}^{0}=\frac{1}{(2 \pi)^{s / 2}} \int_{\mathbb{R}^{s}} e^{i\left(\Phi^{0} \cdot k / \varepsilon\right)}\left(1-\chi\left(\frac{k}{\varepsilon^{\beta}}\right)\right) \widetilde{h}^{0}(k, x) d k .
$$

Отметим, что функция $1-\chi(k)$ финитна; делая в интеграле замену переменной $k=\varepsilon^{\beta} \xi$, получим

$$
h_{B}^{0}=\varepsilon^{s \beta} \frac{1}{(2 \pi)^{s / 2}} \int_{\mathbb{R}^{s}} e^{i\left(\Phi^{0} \cdot \xi / \varepsilon^{1-\beta}\right)}(1-\chi(\xi)) \widetilde{h}^{0}\left(\varepsilon^{\beta} \xi, x\right) d \xi=\varepsilon^{s \beta} H_{B}\left(\frac{\Phi^{0}}{\varepsilon^{1-\beta}}, x, \varepsilon^{\beta}\right),
$$

где $H_{B}(y, x, \varkappa)$ - гладкая функция от всех своих аргументов при $(y, x) \in \mathbb{R}^{3+s}$, $\varkappa \in\left(0, \varkappa_{0}\right)$, причем при каждом фиксированном $\varkappa$ функция $H_{B}$ и все ее производные убывают на бесконечности быстрее любых степеней $|x|,|y|$. Отсюда легко следуют оценки

$$
\left\|h_{B}^{0}\right\|_{W_{2}^{m}} \leqslant \text { const } \cdot \varepsilon^{\beta(s / 2+m)-m+s / 2} .
$$


ЗАмЕчАниЕ 6 . Обозначим через $h_{*}$ вектор, входящий в правые части формул (16). Очевидно, он отличается от построенного в п. 4.1 вектора $h_{1}^{0}+h_{2}^{0}+h_{12}^{0}+h_{21}^{0}$ только отсутствием в интегралах по $k$ срезающей функции $\chi$. Поэтому разность $h_{*}-\left(h_{1}^{0}+\right.$ $\left.h_{2}^{0}+h_{12}^{0}+h_{21}^{0}\right)$ удовлетворяет оценке $(31)$.

\section{5. ОЦЕНКА РАЗРЕШАЮЩЕГО ОПЕРАТОРА}

Для обоснования построенной асимптотики надо получить оценку разрешающего оператора системы (2). Для этого представим ее в виде (4) и запишем соответствующий оператор $\mathcal{L}$ в виде $(5)$. Очевидно, этот оператор представим в виде

$$
\mathcal{L}=\mathcal{L}_{0}+\mathcal{L}_{1}
$$

где

$$
\mathcal{L}_{0}=\left(\begin{array}{cc}
\{W, \cdot\} & 0 \\
0 & \{U, \cdot\}
\end{array}\right),
$$

а $\mathcal{L}_{1}$ - псевдодифференциальный оператор нулевого порядка. Заменим $\mathcal{L}$ на $\mathcal{L}_{0}$ и рассмотрим задачу Коши для параболической системы

$$
\frac{\partial h}{\partial t} h+\mathcal{L}_{0} h=\varepsilon^{2} \mathcal{D} \Delta h,\left.\quad h\right|_{t=0}=h_{0} .
$$

Пусть $G_{0}(x, \xi, t)$ - функция Грина этой задачи Коши, а $G(x, \xi, t, \tau)$ - функция Грина соответствующей задачи с ненулевой правой частью (и нулевым начальным условием). Применяя метод Леви (см., например, [6]) и отслеживая в соответствующих итерациях зависимость от $\varepsilon$ (аналогичные выкладки подробно приведены в работах [7], [8]), получим следующее утверждение.

УтВЕРЖДЕНИЕ 1. На любом конечном промежутке времени справедливы оченки

$$
\begin{aligned}
& \left|G_{0}(x, \xi, t)\right| \leqslant \text { const } \cdot \frac{1}{\varepsilon^{3} t^{3 / 2}} e^{\lambda(x-\xi)^{2} / \varepsilon^{2} t}, \\
& |G(x, \xi, t, \tau)| \leqslant \text { const } \cdot \frac{1}{\varepsilon^{3}(t-\tau)^{3 / 2}} e^{\lambda(x-\xi)^{2} / \varepsilon^{2}(t-\tau)}, \quad \lambda>0 .
\end{aligned}
$$

СлеДСтвиЕ 1. Разрешающий оператор задачи Коши для системы (32) - интегральный оператор, ограниченный на конечном промежутке времени в любом пространстве Соболева.

Рассмотрим теперь полную систему (4).

УтвеРЖДЕНИЕ 2. Разрешающий оператор задачи Коши для системъ (4) ограничен в любом пространстве Соболева.

ДокАЗАТЕЛЬство. Будем считать оператор $\mathcal{L}_{1}$ возмущением и применим метод последовательных приближений. Уравнение для разрешающего оператора стандартным образом сводится к операторному уравнению Вольтерра (см., например, [5], [8]). А именно, пусть $R$ - разрешающий оператор задачи Коши для системы (4), а $R_{0}-$ разрешающий оператор задачи Коши для системы (32). Будем искать $R(t)$ в виде

$$
R(t)=R_{0}(t)+\int_{0}^{t} R_{0}(t-\tau) \Omega(\tau) d \tau .
$$


Учитывая равенства $R_{0}(0)=$ id и

$$
\left(\frac{\partial}{\partial t}+\mathcal{L}_{0}-\varepsilon^{2} \mathcal{D} \Delta\right) R_{0}=0
$$

получим

$$
\Omega(t)+\int_{0}^{t} K(t, \tau) \Omega(\tau) d \tau=F(t),
$$

где $K(t, \tau)=\mathcal{L}_{1} R_{0}(t-\tau), F(t)=-\mathcal{L}_{1} R_{0}(t)$. Можно считать, что нормы обоих последних операторов ограничены в произвольном фиксированном пространстве Соболева одной константой $A$. Из метода последовательных приближений вытекает тогда, что норма $\Omega$ в том же пространстве ограничена при $t \in[0, T]$ константой $A e^{A T}$.

\section{6. ОБОСНОВАНИЕ АСИМПТОТИКИ}

Завершим доказательство теоремы. Пусть $h(x, t, \varepsilon)$ - точное решение системы (4) с начальным условием (3), $h_{A}^{N}-N$-я частичная сумма ряда (18) с достаточно большим $N$, а $h_{A}$ и $h_{B}$ - решения задач Коши с начальными условиями $h_{A}^{0}$ и $h_{0}^{B}$ соответственно, $h_{*}-$ вектор, входящий в правые части формул (16) (старшая часть асимптотики). Очевидно, $h=h_{A}+h_{B}$, причем из оценки (31) и ограниченности разрешающего оператора следует, что

$$
\left\|h_{B}\right\|_{W_{2}^{m}}=O\left(\varepsilon^{\beta(s / 2+m)-m+s / 2}\right) .
$$

Далее из оценки (30) и ограниченности разрешающего оператора следует, что

$$
\left\|h_{A}-h_{A}^{N}\right\|_{W_{2}^{m}}=O\left(\varepsilon^{(N+1-m)(1-\beta)+s / 2}\right),
$$

причем

$$
\left\|h_{*}-h_{A}^{N}\right\|_{W_{2}^{m}}=O\left(\varepsilon^{\beta(s / 2+m)-m+s / 2}\right)
$$

(см. замечание 6). Таким образом, из неравенства треугольника получаем

$$
\left\|h_{*}-h\right\|_{W_{2}^{m}}=O\left(\varepsilon^{\beta(s / 2+m)-m+s / 2}+\varepsilon^{(N+1-m)(1-\beta)+s / 2}\right) .
$$

Зафиксируем произвольное (сколь угодно малое) $\alpha>0$ и выберем $m=3 / 2+\delta$, $\beta=1-\delta, \delta>0$, так, чтобы выполнялось неравенство

$$
\beta\left(\frac{s}{2}+m\right)-m+\frac{s}{2}>1-\alpha .
$$

Убедимся, что такое $\delta$ существует; для этого перепишем левую часть предыдущего неравенства:

$$
(1-\delta)\left(\frac{s}{2}+\left(\frac{3}{2}+\delta\right)\right)-\left(\frac{3}{2}+\delta\right)+\frac{s}{2}=s-\delta^{2}-\delta \frac{s+3}{2} .
$$

При $\delta=0$ правая часть равна $s \geqslant 1$, поэтому при достаточно малых $\delta$ имеем $\beta(s / 2+m)-m+s / 2>1-\alpha$. Теперь зафиксируем $\delta$ и выберем $N$ так, чтобы выполнялось неравенство

$$
(N+1-m)(1-\beta)+\frac{s}{2}>1-\alpha .
$$


Таким образом, при любом $\alpha>0$ для некоторого $m>3 / 2$

$$
\left\|h_{*}-h\right\|_{W_{2}^{m}}=O\left(\varepsilon^{1-\alpha}\right) .
$$

Теперь утверждение теоремы вытекает из теоремы вложения Соболева.

\section{Список литературы}

[1] H. K. Moffatt, Magnetic Field Generation in Electrically Conducting Fluid, Cambridge Univ. Press, Cambridge, 1978.

[2] S. Friedlander, M.M. Vishik, "On stability and instability criteria for magnetohydrodynamics", Chaos, 5:2 (1995), 416-423.

[3] V.V. Kucherenko, "Waves in the linearized system of magnetohydrodynamics", Russ. J. Math. Phys., 17:3 (2010), 272-279.

[4] V. V. Kucherenko, A. Kryvko, "Interaction of Alfven waves in the linearized system of magnetohydrodynamics for an incompressible ideal fluid", Russ. J. Math. Phys., 20:1 (2013), $56-67$.

[5] В. П. Маслов, Операторные методы, Наука, М., 1973.

[6] А. Фридман, Уравнения с частными производными параболического типа, Мир, М., 1968.

[7] А.И. Шафаревич, "Поведение магнитного поля в проводящей жидкости с быстроменяющимся полем скоростей", Докл. РАН, 360:1 (1998), 31-33.

[8] A. I. Esina, A. I. Shafarevich, "Delta-type solutions for a system of induction equations with discontinuous velocity field", Methods Funct. Anal. Topology, 20:1 (2014), 17-33.

Поступила в редакцию 16.05.2016 